2. ASHP drug product bar-code problem reporting center. Bethesda (MD): American Society of Health-System Pharmacists; [cited 2010 Apr 4]. Available from: www.ashp.org/Import/MEMBERCENTER/Sections/ SectionofPharmacyInformaticsandTechnology/Resources/BarCode.aspx

3. Canadian Pharmaceutical Bar Coding Project: joint technical statement on pharmaceutical automated identification and product database requirements. Toronto (ON): Institute for Safe Medication Practices Canada; 2010 [cited 2010 Jun 29]. Available from: www. ismp-canada.org/barcoding/download/CanPharmBarcode_Joint TechnicalStatement.pdf

4. Canadian Pharmaceutical Bar Coding Project. Toronto (ON): Institute for Safe Medication Practices Canada; [cited 2010 Apr 4]. Available from: www.ismp $\neg$ canada.org/barcoding/index.htm

5. What is GTIN? Chicago (IL): Bar Code Graphics Inc; 2009 [cited 2010 May 6]. Available from: www.gtin.info/

6. Bussières JF, Lebel D, Voytenko S, Vaquer G. Développement d'un concept et d'un processus de gestion de l'identité numérique d'un produit en établissement de santé. Can J Hosp Pharm 2009;62(5):406-414.

7. Lebel D, Vaquer G, Forest JM, Bussières JF. Utilisation d'une banque de données photographiques consultées par code-barres pour assister la préparation de seringues orales. Pharm Hosp 2009;44(3):114-124.

8. GS1 application identifiers. Chigaco (IL): Bar Code Graphics Inc; 2010 [cited 2010 May 6]. Available from: www.databar-barcode.info/ application\%2Didentifiers/

Denis Lebel, BPharm, MSC, FCSHP

Assistant Director

Jean-François Bussières, BPharm, MSc, MBA, FCSHP

Director

Department of Pharmacy and Research Unit in Pharmacy Practice

Centre hospitalier universitaire Sainte-Justine

Montréal, Quebec

\section{Explaining the RE-LY Trial}

In questioning the reliability of the RE-LY trial results, Tsang and others ${ }^{1}$ unfortunately based their concerns on a misinterpretation of the data that were published in the original trial report. ${ }^{2}$

The RE-LY trial, which had a study population of 18000 patients, compared 2 doses of dabigatran etexilate with warfarin in the largest randomized controlled trial of antithrombotic therapy for stroke prevention ever performed. The results showed that, relative to warfarin, dabigatran $110 \mathrm{mg}$ twice daily was associated with a similar rate of stroke and a lower rate of life-threatening, intracranial, major, minor, and total bleeding, whereas dabigatran $150 \mathrm{mg}$ twice daily reduced stroke as well as life-threatening intracranial and total bleeding.

The very substantial efficacy and safety benefits of dabigatran over warfarin in the RE-LY trial were associated with a modest excess of myocardial infarction, equivalent to 2 additional myocardial infarctions for every 1000 patients treated. The most likely explanation for this finding is the proven efficacy of warfarin for preventing myocardial infarction. Irrespective of the mechanism, the increase in myocardial infarction was substantially outweighed by the benefits of dabigatran: the reduction in hemorrhagic stroke alone was equivalent to 2.6-2.8 fewer hemorrhagic strokes for every 1000 patients treated. Among patients treated with dabigatran $150 \mathrm{mg}$ bid, major gastrointestinal bleeding was also greater than with warfarin, but this was a subcategory of all major types of bleeding, which was not increased with dabigatran. Thus, contrary to the claim of
Tsang and others, ${ }^{1}$ the RE-LY trial data conclusively demonstrated a net clinical benefit of both doses of dabigatran relative to warfarin.

Tsang and others ${ }^{1}$ criticized the choice of patient population in the RE-LY trial, citing the inclusion of nearly 6000 patients with a CHADS2 score of 0 or 1 , the CHADS(2) being a risk stratification index. Treatment guidelines, however, recommend either warfarin or acetylsalicylic acid for patients with one risk factor for stroke. ${ }^{3}$ Although some patients in the RE-LY trial had a CHADS(2) score of 0 , all patients in the trial had at least one risk factor for stroke, and the results demonstrated a consistent benefit of dabigatran over warfarin in the 6000 patients with a CHADS(2) score of 0 or 1 and in the 12000 patients with a CHADS(2) score of 2 or above. ${ }^{2}$

Tsang and others ${ }^{1}$ challenged the definition of systemic embolism and asked whether patients were screened for systemic emboli. It seems, however, that they have confused systemic arterial embolism with venous thromboembolism. There are no validated methods to screen for systemic embolism, and the trial used a standard definition for symptomatic events that is widely used in randomized controlled trials.

Tsang and others ${ }^{1}$ also raised questions about creatinine monitoring, potential adverse outcomes related to the use of amiodarone and quinidine, and the statistical analysis plan for the study. A creatinine measurement was required for all patients to assess their eligibility to enter the trial. As reported in the original article, ${ }^{2}$ dabigatran had superior efficacy and safety over warfarin, despite lack of routine creatinine screening during the trial, a finding that calls into question the relevance of the concerns raised by the correspondents. Patients who received amiodarone, a drug that can increase blood concentrations of dabigatran by up to $50 \%$, experienced a consistent benefit of dabigatran. Quinidine is rarely used in clinical practice, and very few patients received this drug during the trial. Finally, a per-protocol analysis yielded similar results to those obtained with an intention-to-treat analysis.

References

1. Tsang MP, Tejani A, Kuo I. Are the results of the RE-LY trial reliable? [letter]. Can J Hosp Pharm 2010;63(2):155-156.

2. Connolly SJ, Ezekowitz MD, Yusuf S, Eikelboom JW, Oldgren J, Parekh A, et al.; RE-LY Steering Committee and Investigators. Dabigatran versus warfarin in patients with atrial fibrillation. $N$ Engl J Med 2009; 361(12):1139-1151.

3. Fuster V, Rydén LE, Cannom DS, Crijns HJ, Curtis AB, Ellenbogen KA, et al.; American College of Cardiology/American Heart Association Task Force on Practice Guidelines; European Society of Cardiology Committee for Practice Guidelines; European Heart Rhythm Association; Heart Rhythm Society. ACC/AHA/ESC 2006 Guidelines for the Management of Patients with Atrial Fibrillation: a report of the American College of Cardiology/American Heart Association Task Force on Practice Guidelines and the European Society of Cardiology Committee for Practice Guidelines (Writing Committee to Revise the 2001 Guidelines for the Management of Patients With Atrial Fibrillation): developed in collaboration with the European Heart Rhythm Association and the Heart Rhythm Society. Circulation 2006;114(7):e257-e354.

John W Eikelboom, MBBS

Medicine

Stuart J Connolly, MD

Cardiology

McMaster University

Hamilton, Ontario 


\section{Response from Matthew Tsang, Aaron Tejani, and I fan Kuo}

We thank Drs. Eikelboom and Connolly for responding to our previous letter ${ }^{1}$ and for providing their interpretation of the data from the RE-LY trial. ${ }^{2}$ Although their letter provides useful insights, it does not directly answer our questions and concerns about the trial. We also disagree with their conclusion that "the RE-LY trial data conclusively demonstrated a net clinical benefit of both doses of dabigatran relative to warfarin." As such, we remain convinced that it is inappropriate to imply that dabigatran offers a clear advantage over warfarin for patients with atrial fibrillation, solely on the basis of the published results of the RE-LY trial. ${ }^{2}$ We will attempt to explain our concerns in more detail here.

First, let us explore the issue of net benefit. As stated by Eikelboom and Connolly, dabigatran $110 \mathrm{mg}$ twice daily "was associated with a similar rate of stroke and a lower rate of lifethreatening, intracranial, major, minor, and total bleeding, whereas dabigatran $150 \mathrm{mg}$ twice daily reduced stroke as well as life-threatening intracranial and total bleeding." By definition, any major bleeding episodes, strokes, or systemic emboli are considered serious adverse events. ${ }^{3}$ Simple logic dictates that if dabigatran reduced these outcomes without producing an increase in any other type of serious adverse event, then the number of people experiencing at least one serious adverse event should be lower with either dosage of dabigatran than with warfarin. Conversely, one could conclude that dabigatran results in net harm if the total rate of serious adverse events was the same or higher with dabigatran than with warfarin, despite the reported reduction in bleeding and strokes. In other words, some other serious adverse event might be occurring more frequently with dabigatran, offsetting the reduction in bleeds and strokes. However, it is impossible to do this analysis from the published trial data, as the number of people experiencing at least one fatal or nonfatal serious adverse event (total serious adverse events) was not reported. ${ }^{2}$ We requested these data (and others, as described below) from the authors of the original study, by e-mail correspondence, but received no reply. Compounding this concern is the absolute risk increase of $1.6 \%$ (95\% confidence interval [CI] $1.23 \%-2.00 \% ; p<0.0003$; calculated with Review Manager software version 5) in the number of patients receiving dabigatran $150 \mathrm{mg}$ twice daily who experienced at least one serious adverse event leading to discontinuation of the study drug (see Table 4 of the original trial report ${ }^{2}$ ). The analysis was similar for the $110-\mathrm{mg}$ dose of dabigatran (absolute increase 1.54\%, 95\% CI 1.21\%-1.96\%, $p<0.0005)$. Until we are able to review the data for total serious adverse events (fatal and nonfatal), including the components of the primary composite outcomes, we do not feel confident in drawing conclusions about the net effect of dabigatran relative to warfarin for patients with atrial fibrillation.

We are fully aware that not all investigators report serious adverse events as they should. ${ }^{4}$ For example, some primary outcome events that are by definition serious adverse events are not reported as such. In addition, deaths are sometimes not included in the published analysis of serious adverse events, such that total rates of these events cannot be compared between groups. The only way to know for sure is to seek clarification from the investigators as to how serious adverse events were counted and what exactly was included in the published data on such events. Our attempts to do so, including our previous letter to the $C J H P,{ }^{1}$ have failed.

We agree with Connolly and Eikelboom that patients with at least one risk factor for stroke should be offered either acetylsalicylic acid (ASA) or warfarin. Their letter seems to imply that patients with a CHADS2 score of 0 or 1 (who would have had a lower baseline risk of stroke) could have received ASA. Is it not possible that those patients might have had fewer bleeds and equivalent risk of stroke relative to patients receiving either dabigatran alone or warfarin? If so, is it not also possible that ASA might appear safer than dabigatran, and just as effective, for patients with stroke? We do not feel that Eikelboom and Connelly have provided adequate justification for not offering ASA therapy to these low-risk patients, especially given that they quote recommendations that either warfarin or ASA is considered reasonable. 5

Eikelboom and Connolly suggest that we thought systemic embolism included venous thromboembolic events. In fact, we based our opinion on the meaning of "systemic embolism" provided in the RE-LY trial itself," which states that "systemic embolism was defined as an acute vascular occlusion of an extremity or organ, documented by means of imaging, surgery, or autopsy." This definition does not specify arterial versus venous embolism, and hence venous emboli might have been included in the counts, although the report does not state whether these were screened for or if they were counted only if symptomatic. As such, we were not confused about the terminology and would instead suggest that the text in the trial report did not provide sufficient detail. Even if we ignore the ambiguity related to systemic emboli, there still is a question about how pulmonary emboli were identified: were they symptomatic or asymptomatic? We maintain that if the investigators screened for and counted any asymptomatic embolic event, this information is of debatable clinical importance.

In our previous letter, ${ }^{1}$ we stated that we could not determine, from Table 2 or Table 3 in the main trial report, ${ }^{2}$ the number of people who experienced at least one systemic embolus, fatal stroke, or fatal bleed, nor has this information been provided by Eikelboom and Connolly in their current letter. We are puzzled by the investigators' reluctance to provide these data if they are confident of the robustness of the purported benefit of dabigatran.

We thank Eikelboom and Connolly for confirming that a prespecified per-protocol analysis was performed and that it yielded similar results, confirming our own prediction in this regard. ${ }^{1}$ Such an analysis is recommended by the CONSORT guidelines for reporting randomized controlled trials ${ }^{6}$ but was not mentioned in the original trial report. ${ }^{2}$

In addition, we feel that our concerns about monitoring of creatinine clearance are entirely relevant. The product monograph for dabigatran ${ }^{7}$ suggests that serum levels of the drug will be increased in patients with creatinine clearance of $30-60 \mathrm{~mL} / \mathrm{min}$, leading to increased therapeutic effect and potential for increased adverse drug reactions due to accumulation. Eikelboom and Connelly have confirmed that patients 
with creatinine clearance in this range were included in the trial. Is it not possible that these patients were susceptible to these effects? Figure 2 of the original trial report ${ }^{2}$ shows that the subgroup of patients receiving dabigation $150 \mathrm{mg}$ with creatinine clearance below $50 \mathrm{~mL} / \mathrm{min}$ had a numerically more favourable hazard ratio than those receiving warfarin with creatinine clearance below $50 \mathrm{~mL} / \mathrm{min}$. However, no specific information was provided about the risk of adverse events for the subgroup with creatinine clearance below $50 \mathrm{~mL} / \mathrm{min}$, which might have been higher than for other subgroups. This does not seem to be a major concern, but if it truly is of no importance, why is it mentioned in the product monograph? Further investigation seems warranted to determine the safety of dabigatran for patients with creatinine clearance between 30 and $60 \mathrm{~mL} / \mathrm{min}$. We have similar concerns about the use of amiodarone, in that the rates of adverse events for patients receiving both dabigatran and amiodarone were not reported. This is another area that may require further investigation. Our point about quinidine stemmed from curiosity about the number of patients who might have been using both this drug and dabigatran.

\section{References}

1. Tsang MP, Tejani A, Kuo I. Are the results of the RE-LY trial reliable? [letter]. Can J Hosp Pharm 2010;63(2):155-156.

2. Connolly SJ, Ezekowitz MD, Yusuf S, Eikelboom J, Oldgren J, Parekh A et al.; RE-LY Steering Committee and Investigators. Dabigatran versus warfarin in patients with atrial fibrillation. $N$ Engl $J$ Med 2009;361(12):1139-1151.

3. Serious adverse event analysis: lipid-lowering therapy revisited. Ther Lett [serial on Internet] 2001 Aug-Oct [cited 2009 Sep 17];42:1-2. Available from: www.ti.ubc.ca/PDF/42.PDF
4. International Conference on Harmonisation of Technical Requirements for Registration of Pharmaceuticals for Human Use. ICH harmonised tripartite guideline: guideline for good clinical practice E6, 1996. Available at: www.ich.org/pdfICH/e6.pdf (accessed Aug 2003).

5. Fuster V, Rydén LE, Cannom DS, Crijns HJ, Curtis AB, Ellenbogen KA, et al.; American College of Cardiology/American Heart Association Task Force on Practice Guidelines; European Society of Cardiology Committee for Practice Guidelines; European Heart Rhythm Association; Heart Rhythm Society. ACC/AHA/ESC 2006 guidelines for the management of patients with atrial fibrillation: a report of the American College of Cardiology/American Heart Association Task Force on Practice Guidelines and the European Society of Cardiology Committee for Practice Guidelines (Writing Committee to Revise the 2001 Guidelines for the Management of Patients With Atrial Fibrillation): developed in collaboration with the European Heart Rhythm Association and the Heart Rhythm Society. Circulation 2006;114(7):e257-e354.

6. Schulz KF, Altman DG, Moher D; CONSORT Group. CONSORT 2010 statement: updated guidelines for reporting parallel group randomized trials. Ann Intern Med 2010;152(11):726-732.

7. Pradax [monograph]. In: Repchinksy C, editor. Compendium of pharmaceuticals and specialties [online]. Ottawa (ON): Canadian Pharmacists Association; 2009 [cited 2009 Oct 20]. Available from: http://e-therapeutics.ca. Subscription required to access content.

Matthew P Tsang, BSc(Pharm)

Aaron M Tejani, BSc(Pharm), PharmD, ACPR

I fan Kuo, BSC(Pharm), ACPR, PharmD

Burnaby Hospital

Fraser Health Authority

Burnaby, British Columbia 